\title{
Structural Dynamic Modification Based on Combined Approximations Method
}

\author{
Yuchen Jin ${ }^{1}$, Xiaochen Yang ${ }^{1}$, Rui Feng ${ }^{1}$, Wenjie Zuo ${ }^{1}$ and Guikai Guo ${ }^{{ }^{*}}$ \\ ${ }^{1}$ School of Mechanical and Aerospace Engineering, Jilin University, Changchun, People's Republic of China
}

\begin{abstract}
Structural dynamic modification plays an important role in structural dynamic design. This paper presents an algorithm for dynamic modification of the structure, which is based on the Combined Approximations (CA) approach, sensitivity analysis, Taylor series expansion and the least square method. The feasibility of the algorithm is verified by a numerical example and the results show that the algorithm is accurate enough and easy to be implemented.
\end{abstract}

\section{Introduction}

The structural reanalysis method can accelerate the calculation process of structural dynamic modification and optimization design, and avoid the complete and accurate analysis of the structure repeatedly. This method makes use of the initial information obtained in the last analysis and solution process, and carries on the fast solution calculation, which is an efficient solution method. The Combined Approximation (CA) method is a popular method of reanalysis in recent years. The lower order terms of the binomial series expansion are used to build the basis vectors.

In order to meet the requirements of accuracy and efficiency in the structural analysis, the problem of linear static reanalysis is effectively solved by the CA method which is presented by Kirsch ${ }^{[1]}$. And this method can be well applied to sensitivity analysis, structure layout and topology optimization. Chen ${ }^{[2]}$ improved the CA algorithm, and put forward a general iterative combination approximation (ICA) method so that it can be applied to various types of topology modifications. In the case of changes of degree of freedom, $\mathrm{Wu}^{[3]}$ proposed a method of undetermined coefficient. Levy ${ }^{[4]}$ used modified initial design to calculate the inverse matrix of the modified stiffness matrix. Bae ${ }^{[5-7]}$ calculated the augmented matrix with the combination of SWM formula and series expansion method, and proposed a method for finding the inverse of continuous matrix. In order to improve the accuracy of the solution under the condition of large structural modification, $\mathrm{Chen}^{[8]}$ proposed a matrix perturbation method based on Padé approximate method. A preconditioned Lanczos algorithm is proposed to accelerate convergence of the structural static problems by $\mathrm{Xu}^{[9,}{ }^{10]}$. Zuo ${ }^{[11-13]}$ applied Combined Approximation method to analyze the sensitivity of vibration problem.

CA method is also introduced into modal reanalysis and further developed to solve the problem of time- domain reanalysis. In 2006, $\mathrm{Wu}^{[14]}$ and $\mathrm{Chen}^{[14]}$ introduced the Epsilon method into the modal reanalysis problem. Yang ${ }^{[15]}$ introduced Padé approximation and matrix perturbation method into modal reanalysis, and proposed modal reanalysis method, which increased the variable range of structural parameter modification. For nonlinear problems, its development is not mature, and there are still many problems to be solved, which is the key research direction and development trend in the future.

The background and development of structural reanalysis and dynamic modification are briefly introduced in Sect.1.The formula of structural dynamic modification, which combines sensitivity analysis with Taylor expansion is given in Sect.2. In Sect.3, the algorithm is verified by a numerical example. Finally, the stability and feasibility of the algorithm are discussed in Sect.4.

\section{Formulation and Solution}

\subsection{Modal reanalysis algorithm}

For a typical vibration system, the modal analysis under undamped condition is considered. The eigenvalue problem can be expressed:

$$
\begin{gathered}
K_{0} \Phi_{0}=M_{0} \Phi_{0} \Lambda_{0} \\
K_{0}=U_{0}^{T} U
\end{gathered}
$$

Where $K_{0}$ and $M_{0}$ denotes the total stiffness matrix and mass matrix of the original structure respectively, $U_{0}$ is the upper triangular matrix obtained in modal analysis. When the structure is modified, the eigenvalue problem equation can be written as

$$
K \Phi=M \Phi \Lambda
$$

Therefore, when the structure changes slightly, the modal vector under the new condition can be linearly combined by the following formula:

$$
\varphi=y_{1} r_{1}+y_{2} r_{2}+\ldots \ldots+y_{s} r_{s}=R y
$$

ggk@jlu.edu.cn 
Where $R$ and $y$ are:

$$
\begin{aligned}
& R=\left[r_{1}, r_{2}, \ldots \ldots, r_{s}\right] \\
& y=\left[y_{1}, y_{2}, \ldots . ., y_{s}\right]
\end{aligned}
$$

The above modification realizes the effective reduction of the order of large structures. In order to transfer it into a typical structure, further processing is needed.

$$
R^{T} K R y_{1}=\lambda_{1} R^{T} M R y_{1}
$$

Use the following notation to rewrite equation (5):

$$
\begin{aligned}
& K_{R}=R^{T} K R \\
& M_{R}=R^{T} M R
\end{aligned}
$$

Then, equation (5) can be expressed as the following format:

$$
\mathrm{K}_{R} y_{1}=\lambda_{1} M_{R} y_{1}
$$

In order to improve the accuracy of the mode calculation, the Schmidt orthogonalization and normalization can be used in the subsequent step.

\subsection{Sensitivity analysis and Taylor expansion}

The sensitivity information of the structure is the key to predict the changing trend of the structural response with the structural design variables. It is assumed that the design variable of the structure is $x_{i}$ and the mass and stiffness matrix before modification are $M_{0}$ and $K_{0}$ respectively, while the stiffness and mass matrix after the modification are:

$$
\begin{aligned}
& K=K_{0}+\sum_{i=1}^{m} \Delta K\left(x_{i}\right) \\
& M=M_{0}+\sum_{i=1}^{m} \Delta M\left(x_{i}\right)
\end{aligned}
$$

The response relationships between stiffness, mass matrix and modification of design variables are like this:

$$
\begin{gathered}
K=K_{0}+\sum_{i=1}^{m} \frac{\partial K_{0}}{\partial x_{i}} \Delta x_{i} \\
M=M_{0}+\sum_{i=1}^{m} \frac{\partial M_{0}}{\partial x_{i}} \Delta x_{i}
\end{gathered}
$$

Assume that the design variable modification of the structure is $\Delta x_{i},(i=1,2, \ldots \ldots, m)$, the corresponding eigenvalues are $\lambda_{j}\left(\Delta x_{1}, \Delta x_{2}, \ldots \ldots, \Delta x_{m}\right)$. In order to make the eigenvalue reach the target value after modification, it is important to minimize the absolute value of the phase difference, which means: $\min \left[\lambda-\lambda_{j}\left(\Delta x_{1}, \Delta x_{2}, \ldots \ldots, \Delta x_{m}\right)\right]^{2}$.

The first order Taylor expansion theorem is introduced:

$$
\lambda_{j}\left(\Delta x_{1}, \Delta x_{2}, \ldots \ldots, \Delta x_{m}\right)=\lambda_{j 0}+\sum_{i=1}^{m} \frac{\partial \lambda_{j}}{\partial x_{i}} \Delta x_{i}
$$

Use the least square method to calculate:

$$
\lambda-\lambda_{j 0}=\sum_{i=1}^{m} \frac{\partial \lambda_{j}}{\partial x_{i}} \Delta x_{i}
$$

Now consider the second order Taylor expansion theorem:

$$
\lambda_{j}\left(\Delta x_{1}, \Delta x_{2}, \ldots \ldots, \Delta x_{m}\right)=\sum_{i=1}^{m} \frac{\partial \lambda_{j}}{\partial x_{i}} \Delta x_{i}+\left(\sum_{i=1}^{m} \Delta x_{i} \frac{\partial}{\partial x_{i}}\right)^{2} \lambda_{j}
$$

The calculation is performed using the least square method:

$$
\lambda-\lambda_{j}=\sum_{i=1}^{m} \frac{\partial \lambda_{j}}{\partial x_{i}} \Delta x_{i}+\left(\sum_{i=1}^{m} \Delta x_{i} \frac{\partial}{\partial x_{i}}\right)^{2} \lambda_{j}
$$

Take any binary function $\lambda(x, y)$, differential variable is $t$, the differential equation as shown below can be obtained:

$$
\begin{gathered}
\frac{\partial \lambda}{\partial x}=\frac{\lambda(x+t, y)-\lambda(x-t, y)}{2 t} \\
\frac{\partial \lambda}{\partial y}=\frac{\lambda(x, y+t)-\lambda(x, y-t)}{2 t} \\
\frac{\partial^{2} \lambda}{\partial x^{2}}=\frac{\lambda(x+t, y)-\lambda(x-t, y)-2 \lambda(x, y)}{t^{2}} \\
\frac{\partial^{2} \lambda}{\partial y^{2}}=\frac{\lambda(x, y+t)-\lambda(x, y-t)-2 \lambda(x, y)}{t^{2}} \\
\frac{\partial^{2} \lambda}{\partial x \partial y}=\frac{\lambda(x+t, y-t)-\lambda(x-t, y+t)}{4 t^{2}} \\
-\frac{\lambda(x+t, y+t)-\lambda(x-t, y-t)}{4 t^{2}}
\end{gathered}
$$

According to the above differential equation, we can calculate the sensitivity information with Taylor expansion. Approximate function expression between the design variables and the responses is founded.

\section{Numerical Example}

In order to illustrate the formula effectively, a car door modification example is considered. Choose "PSHELL" as Card Image in Hypermesh and the rigid material parameters of this car door are:

$$
\begin{aligned}
& \rho=7.85 e^{-9} \\
& \mu=0.3 \\
& E=210000
\end{aligned}
$$

The working conditions of the door include two types: the boundary condition and the load condition. For displacement boundary conditions, this example imposes complete constraints on the original model hinges and door rings. The concentrated load is applied to the lower part of the window. (Fig.1).

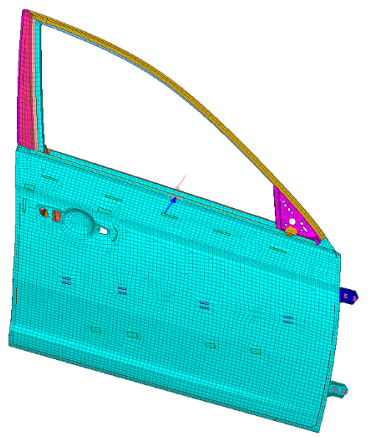

Fig. 1. Load and restraint

In order to simplify the calculation, the grid of door 
model is expanded, and the door model is shown in Fig.2

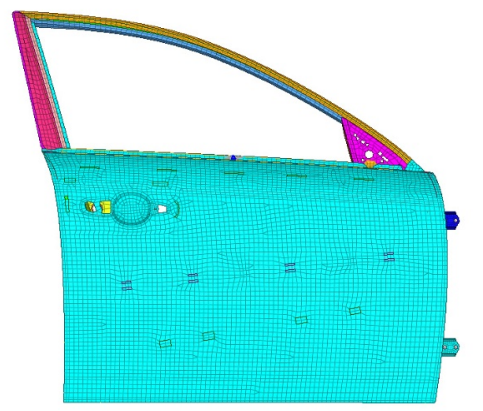

Fig. 2. Finite element model of a car door

In this example, there are two different design variables in the following figure: one is the outer plate of the vehicle, the thickness of the outer plate is defined as $x_{1}$. The second is the reinforcing beam on the inner side

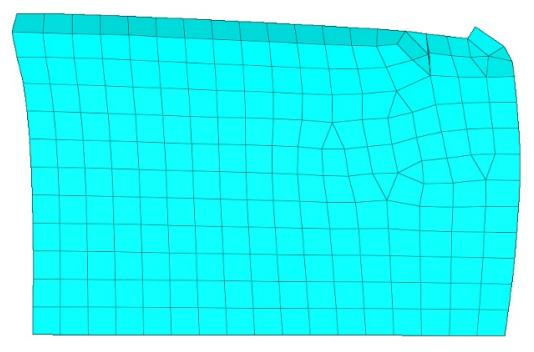

Fig. 4. Door outer plate

The differential variable $t \leq 0.01 \mathrm{~mm}$ is selected and meanwhile the design variables are also modified. The stiffness displacement and the first third order natural frequencies are solved by Hypermesh and Matlab respectively with the required first and second order sensitivity information is obtained in the difference formula. The first order Taylor expansion is used and a certain simplification operation is carried out.

The Taylor expansion of the first natural frequency is:

$$
\omega_{1}=6.55+0.005\left(x_{1}-0.7\right)-0.145\left(x_{2}-1.6\right)
$$

As for the second and third natural frequencies:

$$
\begin{aligned}
& \omega_{2}=15.35+3.13\left(x_{1}-1.6\right)-7.47\left(x_{2}-1.6\right) \\
& \omega_{3}=23.48+24.02\left(x_{1}-0.7\right)+0.056\left(x_{2}-1.6\right)
\end{aligned}
$$

Now use Taylor expansion on the stiffness displacement of the maximum stress point, the result can be written as:

$$
d=0.978324-2.9343\left(x_{1}-0.7\right)-0.0022\left(x_{2}-1.6\right)
$$

Use the same expansion on the overall quality of the model:

$$
m=12.8583+5.8875\left(x_{1}-0.7\right)+0.785\left(x_{2}-1.6\right)
$$

The second order Taylor expansion is used and some simplification operations are carried out.:

The first order natural frequency is: and Fig.3.

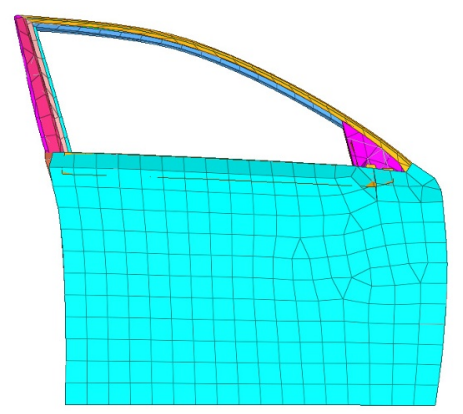

Fig. 3. Simplified model

of the door, and the thickness of the reinforcing beam is defined as $x_{2}$. (Fig.4 and Fig.5)

Fig. 5. Reinforcement beam

$$
\begin{aligned}
\omega_{1}=6.55 & +0.005\left(x_{1}-0.7\right)-0.145\left(x_{2}-1.6\right)+7.1\left(x_{1}-0.7\right)^{2} \\
& +3.66\left(x_{2}-1.6\right)^{2}+0.2\left(x_{1}-0.7\right)\left(x_{2}-1.6\right)
\end{aligned}
$$

Also, the second order Taylor expansions of the second and third order natural frequency are:

$$
\begin{aligned}
\omega_{2}=15.35 & +3.13\left(x_{1}-1.6\right)-7.47\left(x_{2}-1.6\right)+49.7\left(x_{1}-0.7\right)^{2} \\
& +806\left(x_{2}-1.6\right)^{2}+33\left(x_{1}-0.7\right)\left(x_{2}-1.6\right) \\
\omega_{3}=23.48 & +24.02\left(x_{1}-0.7\right)+0.056\left(x_{2}-1.6\right)+2259\left(x_{1}-0.7\right)^{2} \\
& +0.11\left(x_{2}-1.6\right)^{2}-0.51\left(x_{1}-0.7\right)\left(x_{2}-1.6\right)
\end{aligned}
$$

Second order Taylor expansion of the stiffness displacement of the maximum stress point is:

$$
\begin{gathered}
d=0.978324-2.9343\left(x_{1}-0.7\right)-0.0022\left(x_{2}-1.6\right) \\
-14\left(x_{1}-0.7\right)^{2}+0.51\left(x_{1}-0.7\right)\left(x_{2}-1.6\right)
\end{gathered}
$$

According to the requirement of practical engineering application, the constraints imposed in this example are expressed as:

$$
\begin{gathered}
0.5 \mathrm{~mm}<x_{1}<1.5 \mathrm{~mm} \\
1 \mathrm{~mm}<x_{2}<2 \mathrm{~mm}
\end{gathered}
$$

Define the first three orders of the natural frequencies as objectives:

$$
\begin{aligned}
& \omega_{1}=7 \\
& \omega_{2}=16 \\
& \omega_{3}=24
\end{aligned}
$$

The result of the optimization using Taylor expansion as a response can be made. Comparing the design variable 
value with the response value obtained by Lanczos algorithm and ANSYS.

Table1 Comparison of first order Taylor's modification results

\begin{tabular}{lllllll}
\hline & $x_{1} \mathrm{~mm}$ & $x_{2} \mathrm{~mm}$ & $\omega_{1} \mathrm{~Hz}$ & $\omega_{2} \mathrm{~Hz}$ & $\omega_{3} \mathrm{~Hz}$ & $d \mathrm{~mm}$ \\
\hline Lanczos & 0.7259 & 1.4056 & 6.6604 & 15.3904 & 24.0442 & 0.9028 \\
Ansys & 0.7259 & 1.4056 & 6.7070 & 13.9370 & 23.9922 & 0.9073 \\
Error & & & $1.9560 \%$ & $9.4450 \%$ & $0.2160 \%$ & $0.4980 \%$ \\
\hline
\end{tabular}

Table2 Comparison of second order Taylor's modification results

\begin{tabular}{lcccccc}
\hline & $x_{1} \mathrm{~mm}$ & $x_{2} \mathrm{~mm}$ & $\omega_{1} \mathrm{~Hz}$ & $\omega_{2} \mathrm{~Hz}$ & $\omega_{3} \mathrm{~Hz}$ & $d \mathrm{~mm}$ \\
\hline Lanczos & 0.7185 & 1.4614 & 6.5499 & 15.3393 & 23.9388 & 0.9209 \\
Ansys & 0.7185 & 1.4614 & 6.7906 & 13.9260 & 23.8280 & 0.9267 \\
Error & & & $3.675 \%$ & $9.214 \%$ & $0.471 \%$ & $0.63 \%$ \\
\hline
\end{tabular}

By comparing the first order Taylor with the second order Taylor, it can be seen that when the structural modification is small, both the first and second order Taylor can meet the requirements of dynamic modification.

Then CA algorithm is used, and the first and second

Table 3 Comparison of first order Taylor errors in CA method

\begin{tabular}{lcccccc}
\hline & $x_{1} \mathrm{~mm}$ & $x_{2} \mathrm{~mm}$ & $\omega_{1} \mathrm{~Hz}$ & $\omega_{2} \mathrm{~Hz}$ & $\omega_{3} \mathrm{~Hz}$ & $d \mathrm{~mm}$ \\
\hline Lanczos & 0.7121 & 1.5051 & 6.5638 & 16.0987 & 23.7797 & 0.9396 \\
Abaqus & 0.7121 & 1.5051 & 6.7906 & 13.9160 & 23.6960 & 0.9423 \\
Error & & & $3.455 \%$ & $13.558 \%$ & $0.352 \%$ & $0.28 \%$ \\
\hline
\end{tabular}

Table4 Comparison of second order Taylor errors in CA method

\begin{tabular}{lcccccc}
\hline & $x_{1} \mathrm{~mm}$ & $x_{2} \mathrm{~mm}$ & $\omega_{1} \mathrm{~Hz}$ & $\omega_{2} \mathrm{~Hz}$ & $\omega_{3} \mathrm{~Hz}$ & $d \mathrm{~mm}$ \\
\hline Lanczos & 0.7033 & 1.5750 & 6.5560 & 16.0486 & 23.5825 & 0.9689 \\
Abaqus & 0.7033 & 1.5750 & 6.7906 & 13.9010 & 23.4830 & 0.9688 \\
Error & & & $3.578 \%$ & $13.382 \%$ & $0.422 \%$ & $0.01 \%$ \\
\hline
\end{tabular}

Compared with the results of first order Taylor and the second order Taylor formula, it can be seen that a second order Taylor expansion formula can meet the precision in the range of the structural change in the small amplitude. In this condition, the result obtained by the structural reanalysis algorithm is not much different from that of the software calculation result but the calculation amount is effectively reduced, and the calculation efficiency is improved.

\section{Conclusion}

This paper presents a dynamic structural modification algorithm. Through the structural sensitivity information, the response expression between the modification of design variables and the response value is found by using CA algorithm and Taylor expansion formula when modifying the model dynamically. An example is given to verify the accuracy and feasibility of the algorithm, and the purpose of the algorithm is to reduce the amount of computation and improve the computational efficiency.

\section{Acknowledgement}

This work was supported by the National Science
Foundation of China (Grant No. 11502092), the Plan for Education Development of Jilin Province (Grant No. JJKH20190142KJ) and the Plan for Scientific and Technological Development of Jilin Province (Grant No. 20200201272JC).

\section{References}

1. KIRSCH U, PAPALAMBROS P Y. Structural reanalysis for topological modifications - a unified approach [J]. Structural and Multidisciplinary Optimization, 2001, 21(5): 333-44.

2. CHEN S H, YANG Z J. A universal method for structural static reanalysis of topological modifications [J]. International Journal for Numerical Methods in Engineering, 2004, 61(5): 673-86.

3. BAISHENG W, ZHENGGUANG L. Static reanalysis of structures with added degrees of freedom [J]. Communications in Numerical Methods in Engineering, 2006, 22(4): 269-81.

4. LEVY R, KIRSCH U, LIU S. Reanalysis of trusses using modified initial designs [J]. Structural and Multidisciplinary Optimization, 2000, 19(2): 105-12.

5. BAE H-R, GRANDHI $\mathrm{R} V$, CANFIELD $\mathrm{R} A$. Successive matrix inversion method for reanalysis of 
engineering structural systems [J]. AIAA Journal, 2004, 42(8): 1529-35.

6. BAE $\mathrm{H} \mathrm{R}$, GRANDHI $\mathrm{R} \mathrm{V}$, CANFIELD $\mathrm{R} \mathrm{A}$. Accelerated engineering design optimization using successive matrix inversion method [J]. International Journal for Numerical Methods in Engineering, 2006, 66(9): 1361-77.

7. BAE H-R, GRANDHI $\mathrm{R} \mathrm{V}$, CANFIELD $\mathrm{R}$ A. Efficient successive reanalysis technique for engineering structures [J]. AIAA Journal, 2006, 44(8): 1883-9.

8. CHEN $\mathrm{S} H$, YANG $\mathrm{X}$ W, WU B S. Static displacement reanalysis of structures using perturbation and Pade approximation [J]. COMMUNICATIONS IN NUMERICAL METHODS IN ENGINEERING, 2000, 16(2): 75-82.

9. XU TAO C F, YU LAN. New method for structural static reanalysis of topological modifications based on preconditioning Lanczos algorithm $[\mathrm{J}]$. College of Mechanical Science and Engineering,Jilin University, 2007, 37(5): 1214-9.

10. XU T, ZUO W, XU T, et al. An adaptive reanalysis method for genetic algorithm with application to fast truss optimization [J]. Acta Mechanica Sinica, 2010, 26(2): 225-34.

11. ZUO W, XU T, ZHANG H, et al. Fast structural optimization with frequency constraints by genetic algorithm using adaptive eigenvalue reanalysis methods [J]. Structural and Multidisciplinary Optimization, 2011, 43(6): 799-810.

12. ZUO W, YU Z, ZHAO S, et al. A hybrid Fox and Kirsch's reduced basis method for structural static reanalysis [J]. Structural and Multidisciplinary Optimization, 2012, 46(2): 261-72.

13. ZUO W, HUANG K, BAI J, et al. Sensitivity reanalysis of vibration problem using combined approximations method [J]. Structural and Multidisciplinary Optimization, 2017, 55(4): 1399405.

14. WU B, LI Z. Static reanalysis of structures with added degrees of freedom [J]. Communications in Numerical Methods in Engineering, 2006, 22(4): 26981.

15. YANG Z, CHEN X, KELLY R. An Adaptive Static Reanalysis Method for Structural Modifications Using Epsilon Algorithm, F 2009]. IEEE. 